\title{
Burnout na clínica psiquiátrica: relato de um caso
}

\author{
Isabela Vieira* \\ Andréia Ramos** \\ Dulcéa Martins ${ }^{\star * \star}$ \\ Erika Bucasio**** \\ Ana Maria Benevides-Pereira\# \\ Ivan Figueira\#\# \\ Sílvia Jardim\#\#\#
}

\section{INTRODUÇÃO}

A associação entre condições de trabalho e ocorrência de doenças físicas e transtornos mentais vem sendo mais estudada a partir da segunda metade do século $X X^{1}$, mas 0 reconhecimento clínico de tal relação é pequeno ${ }^{2}$. O burnout, ou síndrome do

* Médica psiquiatra, Programa de Atenção em Saúde Mental do Trabalhador (PRASMET) e Programa de Transtornos Mentais Relacionados ao Estresse, Instituto de Psiquiatria - Universidade Federal do Rio de Janeiro (IPUB-UFRJ), Rio de Janeiro, RJ

** Doutora em Psiquiatria, PRASMET, IPUB-UFRJ, Rio de Janeiro, RJ.

${ }^{\star * *}$ Assistente social. Doutoranda, Escola de Serviço Social, Universidade Federal do Rio de Janeiro (UFRJ), Rio de Janeiro, RJ.

**** Médica psiquiatra, PRASMET e Programa de Transtornos Mentais Relacionados ao Estresse, IPUB-UFRJ, Rio de Janeiro, RJ. Mestranda, IPUB-UFRJ, Rio de Janeiro, RJ.

\# Doutora em Psicologia. Líder, Grupo de Estudos e Pesquisas sobre Estresse e Burnout (GEPEB), Universidade Estadual de Maringá (UEM) Maringá, PR. Professora associada, Departamento de Psicologia, UEM, Maringá, PR.

\#\# Coordenador, Programa de Transtornos Mentais Relacionados ao Estresse, IPUB-UFRJ, Rio de Janeiro, RJ. Professor adjunto, Departamento de Psiquiatria e Saúde Mental, UFRJ, Rio de Janeiro, RJ.

\#\#\# Coordenadora, PRASMET, IPUB-UFRJ, Rio de Janeiro, RJ. Psiquiatra, IPUB-UFRJ, Rio de Janeiro, RJ. esgotamento profissional, tem sido alvo de estudos de prevalência ${ }^{3}$, análises da validade de constructo ${ }^{4}$, identificação de fatores de risco ou de proteção ${ }^{5}$ e objeto de matérias na imprensa. Na literatura médica, tem ocupado espaço fora da psiquiatria, particularmente na medicina ocupacional, psicossomática e clínica médica.

Estudos de prevalência com profissionais de saúde mostram taxas de burnout variando entre 30 e $47 \%^{6,7}$. A taxa de burnout na população de trabalhadores da Finlândia chegou a $27,6 \%^{8}$. No Brasil, a ocorrência se encontra na faixa de $10 \% 9$.

Por definição, burnout é uma condição de sofrimento psíquico relacionada ao trabalho. Está associado com alterações fisiológicas decorrentes do estresse ${ }^{10}$ (maior risco de infecções, alterações neuroendócrinas do eixo hipotalâmico-hipofisário-adrenal, hiperlipidemia, hiperglicemia e aumento do risco cardiovascular), abuso de álcool e substâncias ${ }^{11}$, risco de suicídio ${ }^{12}$ e transtornos ansiosos e depressivos ${ }^{13,14}$, além de implicações socioeconômicas (absenteísmo, abandono de especialidade, queda de produtividade ${ }^{14,15}$ ).

Recebido em 13/03/2006. Aceito em 06/10/2006. 
Entretanto, não consta nas classificações psiquiátricas.

Freudenberger ${ }^{16,17}$, em 1974 , descreveu o burnout como um "incêndio interno" resultante da tensão produzida pela vida moderna, afetando negativamente a relação subjetiva com o trabalho. Segundo Maslach et al. ${ }^{18}$, o burnout é uma síndrome psicológica resultante de estressores interpessoais crônicos no trabalho e caracteriza-se por: exaustão emocional, despersonalização (ou ceticismo) e diminuição da realização pessoal (ou eficácia profissional). A exaustão emocional (EE) caracteriza-se por fadiga intensa, falta de forças para enfrentar o dia de trabalho e sensação de estar sendo exigido além de seus limites emocionais. A despersonalização (DE) caracteriza-se por distanciamento emocional e indiferença em relação ao trabalho ou aos usuários do serviço. A diminuição da realização pessoal (RP) se expressa como falta de perspectivas para o futuro, frustração e sentimentos de incompetência e fracasso. Também são comuns sintomas como insônia, ansiedade, dificuldade de concentração, alterações de apetite, irritabilidade e desânimo.

$O$ instrumento mais utilizado para o diagnóstico de burnout é o Maslach Burnout Inventory $(\mathrm{MBI})^{19}$. Possui três versões aplicáveis a categorias profissionais específicas: MBI-HSS (Human Services Survey), para as áreas de saúde/cuidadores ou serviços humanos/sociais; MBI-ES (Educator's Survey), para educadores; e MBI-GS (General Survey), para profissionais que não estejam necessariamente em contato direto com o público-alvo do serviço. É auto-aplicável e avalia as três dimensões do burnout (EE, DE e RP). No Brasil, até o momento, só estão publicadas adaptações para o português das versões MBI-HSS e MBI-ES ${ }^{20}$.

É típica a história pessoal de grande envolvimento no trabalho, visto como prioridade de vida ou uma missão. Entretanto, os fatores relacionados à organização do trabalho (divisão do trabalho, tempos, ritmos e duração das jornadas, remuneração e estrutura hierárquica) ${ }^{21}$ são considerados preponderantes na determinação da síndrome ${ }^{22}$. Pesquisas recentes vêm ressaltando a importância da personalidade e do temperamento como fatores de risco para burnout ${ }^{23}$.

A síndrome do esgotamento profissional integra a Lista de Doenças Profissionais e Relacionadas ao Trabalho (Ministério da Saúde, Portaria no 1339/1999) ${ }^{24}$. Está classificada sob o código Z73.0 (Classificação Internacional de
Doenças, 10a revisão - CID-10), como problema que leva ao contato com serviços de saúde.

Apresentamos um caso que preenche os critérios diagnósticos para burnout e depressão. Discute-se, então, a diferença entre burnout e depressão e o status nosológico do constructo burnout.

\section{RELATO DE CASO}

A., 50 anos, casado, técnico em telecomunicações, funcionário de empresa de telefonia há 28 anos. Seus problemas começaram em 1996, com sucessivas mudanças administrativas: foi transferido de unidade duas vezes e assumiu, sem consulta prévia, posto de gerência, aumentando suas atribuições, enquanto reduzia-se o efetivo de pessoal. Suas novas tarefas incluíam a demissão de funcionários. Para aprender o novo serviço, passou a trabalhar até mais tarde nos fins de semana. Começou a sentir-se muito cansado fisicamente, ansioso, tenso e insone. Após a privatização da empresa, instalou-se o processo de reestruturação produtiva, com demissões em massa e expansão dos serviços. Os novos contratados não estavam suficientemente qualificados para as funções, exigindo maior esforço na tarefa de supervisão. Havia sucessivas "mudanças de diretriz" ("mandavam a gente fazer tudo de um jeito, e no dia seguinte não era mais nada daquilo, o trabalho era jogado fora"), além das ameaças de demissão, da desmoralização dos funcionários e das exigências cada vez maiores de rendimento ("quando a meta não era alcançada, era porque éramos incompetentes; quando se conseguia, deveríamos ter nos esforçado mais para superá-la"). Além do cansaço físico, sentia-se exigido além do seu limite emocional. Pensar em trabalho deixava-o irritado e impaciente, ao contrário do que sempre foi (considerava-o como prioridade, fonte de satisfação pessoal e orgulho). Passou a apresentar, além da ansiedade, tristeza profunda, falta de prazer nas atividades, dificuldade em tomar decisões, perda de apetite e de peso (cerca de $14 \mathrm{~kg}$ em 7 meses), "brancos" de memória, desesperança, sentimento de desvalorização pessoal e vontade de morrer. Foi, então, afastado de suas atividades laborativas, e iniciou tratamento psiquiátrico em 2000. Fez uso de diversas associações: tioridazina $10-30 \mathrm{mg} / \mathrm{dia}$, cloxazolam $2 \mathrm{mg} / \mathrm{dia}$, sulpirida 300-600 mg/dia, biperideno 2-4 mg/dia, nortriptilina $25-75 \mathrm{mg} /$ 
dia. Foi encaminhado ao Instituto de Psiquiatria da Universidade Federal do Rio de Janeiro (IPUB) após 2 anos de tratamento privado. Vinha em uso de oxcarbazepina $600 \mathrm{mg} / \mathrm{dia}$, clonazepam $4 \mathrm{mg} /$ dia e levomepromazina 50 $\mathrm{mg} /$ dia, que foram então substituídos por imipramina 75-150 mg/dia, clorpromazina 100 $\mathrm{mg} /$ dia e diazepam $10 \mathrm{mg} /$ dia (esquema atual). Evoluiu com melhora importante da ansiedade e da insônia, melhora parcial dos sintomas depressivos e intensa dificuldade de lidar com situações referentes ao trabalho. Foi aposentado por invalidez 1 ano após sua admissão no ambulatório do IPUB.

\section{DISCUSSÃO}

O caso descrito apresenta vários dos fatores relativos à organização do trabalho considerados por Maslach et al. como determinantes do burnout $^{18}$ - sobrecarga, insegurança em relação às tarefas, falta de condições para exercer o trabalho, insegurança quanto à permanência no emprego, falta de suporte da equipe/chefia, sentimento de desmoralização pessoal no ambiente de trabalho, sentimento de injustiça. Os fatores pessoais de dedicação ao trabalho também estão presentes. Os sintomas emocionais desenvolvidos pelo paciente correspondem às três dimensões características da síndrome de burnout.

O paciente apresenta também depressão. Embora apareçam associados com freqüência, vários estudos mostram que burnout e depressão são conceitualmente diferentes. Segundo Freudenberger, o "estado depressivo" presente no burnout seria temporário e orientado para uma situação precisa na vida da pessoa (no caso, o trabalho), além do que não estaria presente o sentimento de culpa, característico da depressão ${ }^{17}$; também para Maslach, o burnout afetaria somente o campo profissional, enquanto que a depressão atingiria todas as áreas da vida do indivíduo ${ }^{18}$. Brenninkmeyer ${ }^{25}$ sistematiza essas diferenças. Comparados a indivíduos deprimidos, os que têm burnout: 1) aparentam mais vitalidade e são mais capazes de obter prazer nas atividades; 2) raramente apresentam perda de peso, retardo psicomotor ou ideação suicida; 3) têm sentimentos de culpa mais realistas, se os têm; 4) atribuem sua indecisão e inatividade à fadiga (e não à própria doença); e 5) apresentam mais freqüentemente insônia inicial, em vez de terminal (como na depressão). A natureza da associação burnout/depressão ainda não é bem conhecida: pode se dever a antecedentes etiológicos comuns (ligados ao estresse crônico ou a fatores de personalidade, como traços neuróticos, por exemplo), podendo ser o burnout uma fase (ou um precursor) no desenvolvimento de um transtorno depressivo ${ }^{8}$. lacovides ${ }^{26}$ sugere que burnout e depressão poderiam compartilhar várias características "qualitativas", especialmente nas formas mais graves de burnout, propondo que sejam aplicados os dois diagnósticos em certos casos, tais como: aqueles em que haja maior grau de disfunção no trabalho do que de sintomatologia depressiva, início da disfunção antes do início da depressão maior ou a existência de uma atitude negativa em relação à profissão que não pode ser explicada como uma manifestação da depressão.

A sobreposição, no caso apresentado, da síndrome de burnout com depressão leva-nos a duas hipóteses: 1) a demora no reconhecimento do problema poderia ter resultado no desenvolvimento de uma complicação (a depressão); ou 2) tal caso pertenceria a um subtipo de pacientes com maior vulnerabilidade ao desenvolvimento de burnout e que estaria associado com maior gravidade do quadro e semelhança fenotípica com depressão (de acordo com o estudo de lacovides) ${ }^{27}$. Ahola et al. ${ }^{8}$ sugerem que "ao lidar-se com população de trabalhadores, é recomendável aferir-se tanto a existência de burnout quanto de depressão".

Este caso é um exemplo de que o burnout parece estar relacionado não a profissões específicas, e sim à maneira como se organiza o trabalho, independente da atividade exercida ${ }^{18,28}$. O determinante fundamental parece ser a impossibilidade encontrada por pessoas profundamente empenhadas em atingir um ideal (aqui representado pelo engajamento no trabalho) de realizar tal meta, impossibilidade esta também determinada pelas características da organização do trabalho.

A síndrome de burnout é um constructo em investigação. Segundo Maslach, o burnout não é uma síndrome clínica, e sim um diagnóstico de situação de trabalho ${ }^{18}$. Será que os casos que preenchem os critérios para burnout, segundo a escala de Maslach, na clínica psiquiátrica seriam casos a mais de depressão? Como estabelecer, então, a relação de tais casos com a organização do trabalho?

Um relato de caso não pode responder a perguntas que somente estudos prospectivos poderão, mas abre a discussão sobre a síndrome de burnout na clínica e nosologia psiquiátricas. 


\section{REFERÊNCIAS}

1. Jardim SR, Glina DMR. O diagnóstico dos transtornos mentais relacionados ao trabalho. In: Glina DMR, Rocha LE, orgs. Saúde mental no trabalho: desafios e soluções. São Paulo: VK; 2000. p. 17-52.

2. Harber $P$, Mullin M, Merz B, Tarazi M. Frequency of occupational health concerns in general clinics. J Occup Environ Med. 2001;43(11):939-45.

3. Kirwan M, Armstrong D. Investigation of burnout in a sample of British general practitioners. Br J Gen Pract. 1995;45(394): 259-60.

4. Pierce CM, Molloy GN. The construct validity of the Maslach Burnout Inventory: some data from down under. Psychol Rep. 1989;65(3 Pt 2):1340-2.

5. Aries M, Ritter IZ. Nurses with and without burnout: a comparison. Results of a quantitative longitudinal study and a qualitative depth study. Pflege. 1999;12(2):83-8.

6. Caballero Martin M, Bermejo Fernandez F, Nieto Gómez R, Caballero Martinez F. Prevalence and factors associated with burnout in a health area. Aten Primaria. 2001;27(5):313-7.

7. Atance Martinez JC. Epidemiologic aspects of the burnout syndrome in hospital personnel. Rev Esp Salud Publica. 1997;71(3):293-303.

8. Ahola K, Honkonen T, Isometsä E, Kalimo R, Nykyri E, Aromaa $A$, et al. The relationship between job-related burnout and depressive disorders - results from the Finnish Health 2000 Study. J Affect Disord. 2005;88(1):55-62.

9. Benevides-Pereira AMT, org. Burnout: quando o trabalho ameaça o bem-estar do trabalhador. São Paulo: Casa do Psicólogo; 2002.

10. Shirom A, Melamed S, Toker S, Berliner S, Shapira I. Burnout, mental and physical Health: a review of the evidence and a proposed explanatory model. Int Rev Ind Organ Psychol. 2005;20:269-309.

11. Cunradi CB, Greiner BA, Ragland DR, Fisher JM. Burnout and alcohol problems among urban operators in San Francisco. Addict Behav. 2003;28(1):91-109.

12. Samuelsson M, Gustavsson JP, Petterson IL, Arnetz $\mathrm{B}$, Asberg M. Suicidal feelings and work environment in psychiatric nursing personnel. Soc Psychiatry Psychiatr Epidemiol. 1997;32(7):391-7.

13. Soares JJ, Jablonska B. Psychosocial experiences among primary care patients with and without musculoskeletal pain. Eur J Pain. 2004;8(1):79-89.

14. Felton JS. Burnout as a clinical entity - its importance in health care workers. Occup Med (Lond). 1998;48(4):237-50.

15. Barrett L, Yates P. Oncology/haematology nurses: a study of job satisfaction, burnout and intention to leave the specialty. Aust Health Rev. 2002;25(3):109-21.

16. Freudenberger HJ. Staff burnout. J Soc Issues. 1974;30:159-65.

17. Freudenberger HJ, Richelson G. L'épuisement professionel: la brûlure interne. Ottawa: Ed Gaëtan Morin; 1987.

18. Maslach C, Schaufeli WB, Leiter MP. Job burnout. Annu Rev Psychol. 2001;52:397-422.

19. Maslach C, Jackson SE, Leiter MP. Maslach Burnout inventory manual. 3rd ed. Palo Alto, CA: Consulting Psychologist's Press; 1996.

20. Roazzi A, Carvalho AD, Guimarães PV. Análise da estrutura de similaridade de burnout: validação da escala Maslach Burnout Inventory em professores. In: Anais do V Encontro Mineiro de Avaliação Psicológica: Teorização e Prática. VIII Conferência Internacional de Avaliação Psicológica - Formas e Contexto e V
Encontro Mineiro de Avaliação Psicológica: Teorização e Prática. Belo Horizonte: Pontifícia Universidade Católica de Minas Gerais; 2000.

21. Jardim SR, Silva Filho JF. O desemprego do trabalho na saúde mental: campo e poder do diagnóstico psiquiátrico na assistência aos trabalhadores. In: Venâncio AT, Cavalcanti MT, orgs. Saúde mental: campo, saberes e discursos. Rio de Janeiro: IPUB/ CUCA; 2001. p. 193-214.

22. Thomsen S, Soares J, Nolan P, Dallender J, Arnetz B. Feelings of professional fulfillment and exhaustion in mental health personnel: the importance of organisational and individual factors. Psychother Psychosom. 1999;68(3):157-64.

23. Thoresen CJ, Kaplan SA, Barsky AP, Warren CR, de Chermont $\mathrm{K}$. The affective underpinnings of job perceptions and attitudes: a meta-analytic review and integration. Psychol Bull. 2003;129(6):914-45.

24. Brasil, Ministério da Saúde. Doenças relacionadas ao trabalho: manual de procedimentos para os serviços de saúde. Brasília: Ministério da Saúde; 2001.

25. Brenninkmeyer V, Van Yperen NW, Buunk BP. Burnout and depression are not identical twins: is decline of superiority a distinguishing feature? Pers Individ Dif. 2001;30(5):873-80.

26. lacovides A, Fountoulakis KN, Kaprinis S, Kaprinis G. The relationship between job stress, burnout and clinical depression. J Affect Disord. 2003;75(3):20921.

27. lacovides A, Fountoulakis KN, Moysidou C, lerodiakonou C. Burnout in nursing staff: a clinical syndrome rather than a psychological reaction? Gen Hosp Psychiatry. 1999;19(6):419-28.

28. Silveira NM, Vasconcellos SJL, Cruz LP, Kiles RF, Silva TP, Castilhos DG, et al. Avaliação de burnout em uma amostra de policiais civis. Rev Psiquiatr RS. 2005;27(2):159-63.

\section{RESUMO}

A síndrome de burnout, identificada na década de 1970, caracteriza-se por uma tríade de dimensões (exaustão emocional, despersonalização e redução da realização pessoal) e é uma condição relacionada à organização do trabalho. Entretanto, não consta nas classificações psiquiátricas. $O$ artigo apresenta o conceito de burnout, estabelece o seu atual status nosológico e introduz uma breve discussão sobre a diferença entre burnout e depressão, a partir do relato de um caso atendido no Instituto de Psiquiatria da Universidade Federal do Rio de Janeiro.

Descritores: Burnout, depressão, diagnóstico, organização do trabalho.

\section{ABSTRACT}

The burnout syndrome was first described in the 1970's and is characterized by three dimensions (emotional exhaustion, depersonalization and reduced personal accomplishment). Burnout is a work organization-related health condition. Nevertheless, it is not part of any psychiatric classification. This article presents the concept of burnout, establishes its 
nosological status and introduces a brief discussion on the difference between burnout and depression, based on the report of a clinical case treated at Instituto de Psiquiatria da Universidade Federal do Rio de Janeiro.

Keywords: Burnout, depression, diagnostic, work organization.

Title: Burnout in psychiatric practice: a case report

\section{Correspondência:}

Isabela Vieira

Rua das Laranjeiras, 361/801, Laranjeiras

CEP 22240-005 - Rio de Janeiro, RJ

Tel: (21) 2205.8677, (21) 9977.9980

Fax: (21) 2225.0060

E-mail: isabelamvieira@gmail.com

Copyright (C) Revista de Psiquiatria do Rio Grande do Sul - SPRS 